\title{
Buoyancy-driven flow inside an asymmetrically heated cavity
}

\author{
A. Demou, D.G.E. Grigoriadis, B.J. Geurts
}

\section{Introduction}

Buoyancy-driven flows inside enclosures are in the center of problems related to heat transfer because they can provide a significant insight into the physical mechanisms of heat transfer. Typical examples of such flows include Rayleigh-Bénard convection, differentially heated cavities and partially divided enclosures. In the present study, the buoyancy-driven flow inside an asymmetrically heated closed cavity is investigated and proposed as a benchmark case for future studies to assess the accuracy of simulations and to help in the validation of coarsened turbulence models. Additionally, from an application perspective such a configuration is highly relevant, e.g., in view of its similarity with passive solar systems such as ventilated building facades [1] and Trombe walls [2].

We propose a benchmark study of the flow and heat transfer characteristics inside a closed cuboid cavity with an interior heated wall and a cooled side wall, while all other boundaries are treated as adiabatic. The heated wall is asymmetrically located closer to one side of the cavity, as shown in figure 1. Constant temperature boundary conditions are applied on both the heated and cooled walls.

Results are compared for a wide range of Rayleigh numbers, $R a=10^{5}-3.2 \times$ $10^{9}$, based on the height of the heated wall and its temperature. The effect of $R a$ on the generated heat transfer as characterised by the Nusselt number $N u$ as well as the associated flow patterns in the cavity will be presented.

\footnotetext{
A. Demou · D.G.E. Grigoriadis

University of Cyprus, e-mail: andreas.demou@gmail.com, dimokratisg@gmail.com

B.J. Geurts

University of Twente, e-mail: b.j.geurts@utwente.nl
} 


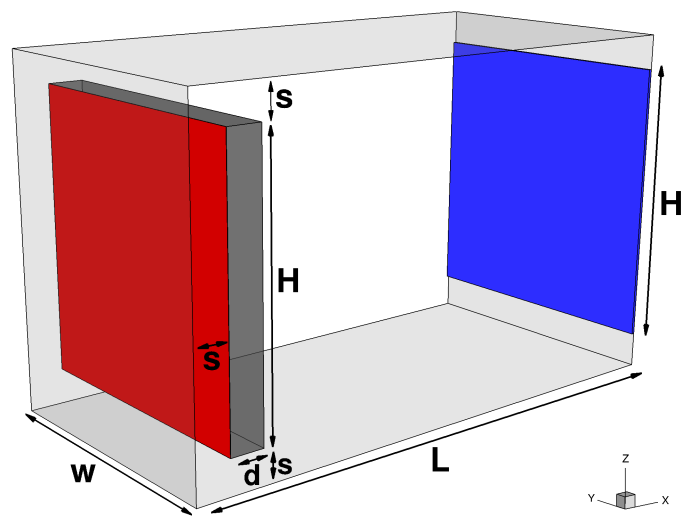

Fig. 1 Geometrical configuration with the heated and cooled surfaces shown with red and blue colour respectively. The figure introduces the characteristic sizes that define the geometry in terms of heights $H, L$ and $w$ as well as thickness $d$ of the hot wall and separation from the walls $s$.

\section{Mathematical and numerical modeling}

\subsection{Mathematical modeling}

Assuming that the flow is incompressible and that the Boussinesq approximation is valid, the governing equations for natural convection take the form,

$$
\begin{array}{r}
\frac{\partial u_{j}}{\partial x_{j}}=0 \\
\frac{\partial u_{i}}{\partial t}+\frac{\partial u_{i} u_{j}}{\partial x_{j}}=-\frac{\partial p}{\partial x_{i}}+\frac{\operatorname{Pr}}{\sqrt{R a}} \frac{\partial^{2} u_{i}}{\partial x_{j} \partial x_{j}}+\operatorname{Pr} \Theta \delta_{i 3} \\
\frac{\partial \Theta}{\partial t}+\frac{\partial \Theta u_{j}}{\partial x_{j}}=\frac{1}{\sqrt{R a}} \frac{\partial^{2} \Theta}{\partial x_{j} \partial x_{j}}
\end{array}
$$

where $i=1,2,3$, represent the $x, y$ and $z$ directions and the respective velocities $u_{i}$ in each direction are denoted hereafter also as $u, v, w$. Gravity acts along the $z$ $\left(=x_{3}\right)$ direction and $P r$ is the Prandtl number. $R a$ represents the Rayleigh number which can be defined with respect to the wall and ambient temperatures $\left(T_{w}\right.$ and $\left.T_{\infty}\right)$ according to,

$$
R a_{\Delta T}=\frac{g \beta\left(T_{w}-T_{\infty}\right) H^{3}}{v \alpha}
$$

In equation $4, \beta$ is the coefficient of volumetric expansion, $v$ the kinematic viscosity, $\alpha$ the thermal diffusivity and $k$ the thermal conductivity of the fluid. Equations 1 to 3 have been non-dimensionalised using the height of the heated wall $H$ as the characteristic length scale, $V_{0}=\alpha \sqrt{R a} / H$ as a velocity scale, $t_{0}=H / V_{0}$ as a time scale and $P_{0}=\rho V_{0}^{2}$ as a pressure scale. The non-dimensional temperature is defined 
as $\Theta=\left(T-T_{\infty}\right) / T_{0}$ where the characteristic temperature scale $T_{0}$ is taken as the temperature difference $\left(T_{w}-T_{\infty}\right)$.

The Nusselt number is defined for all cases with respect to the wall height as $N u=h H / k$, where $h$ is the convection heat transfer coefficient. $N u$ is calculated as the average temperature gradient, normal to the heated wall, i.e.

$$
N u=\left\langle\frac{\partial \Theta}{\partial n}\right\rangle_{w}
$$

\subsection{Numerical methodology}

The numerical method used is based on a second order finite difference method on Cartesian staggered grids utilising a direct pressure solver. Velocities are discretised in space using central differences. For the temperature equation 3, a hybrid linear parabolic approximation (HLPA) scheme was implemented [3]. Time advancement consisted of a fully explicit Adams-Basforth scheme. The presence of obstacles inside the flow is achieved with the use of the Immersed Boundary method [4].

\subsection{Computational parameters}

Results will be presented for a cuboid cavity with $L=2 H$ and a wall spacing equal to the wall thickness, i.e., $s=d=H / 8$ (figure 1). Preliminary simulations revealed independence of the time-averaged isotherms and the two-point correlations when the spanwise length of the domain is larger than $H$. Therefore, the spanwise extent $w$ of the domain is chosen equal to $H$ so that periodic boundary conditions can be applied without suppressing the contained flow structures. For all cases examined, the Prandtl number is set to $P r=0.71$, i.e., the cavity is assumed to be filled with air.

No-slip boundary conditions are used for the velocity field along solid boundaries. The boundary conditions along the heated and cooled walls are specified as constant temperature $\left(\Theta_{w}= \pm 1\right)$. All other boundary surfaces are assumed to be adiabatic.

The grid resolution was carefully selected for each case, so that the near-wall dynamics are properly resolved [5], [6]. For the range of $R a$ numbers considered here, eight to ten points were placed within the thickness of the boundary layers. Table 1 lists the test cases that were studied, along with the resolution used.

\section{Results}

\subsection{Statistical convergence}

Initially, the air adjacent to the heated surface warms up, rises due to buoyancy and 
Table 1 Test cases in 2D and 3D presented, $R a$ numbers and million nodes for each numerical grid used.

\begin{tabular}{llll}
\hline Case & $R a_{\Delta T}$ & Grid & Mnodes \\
\hline T1 & $1.0 \times 10^{5}$ & $56 \times 38(2 D)$ & 0.002 \\
T2 & $1.0 \times 10^{6}$ & $114 \times 82(2 D)$ & 0.009 \\
T3A & $1.0 \times 10^{7}$ & $206 \times 164(2 D)$ & 0.03 \\
T3B & $1.0 \times 10^{7}$ & $206 \times 128 \times 164$ & 4.3 \\
T4A & $1.0 \times 10^{8}$ & $380 \times 298(2 D)$ & 0.1 \\
T4B & $1.0 \times 10^{8}$ & $380 \times 128 \times 298$ & 15 \\
T5A & $1.0 \times 10^{9}$ & $764 \times 558(2 D)$ & 0.4 \\
T5B & $1.0 \times 10^{9}$ & $764 \times 128 \times 558$ & 54.6 \\
T6A & $3.2 \times 10^{9}$ & $764 \times 558(2 D)$ & 0.4 \\
T6B & $3.2 \times 10^{9}$ & $764 \times 160 \times 558$ & 68.2 \\
\hline
\end{tabular}

hits the roof of the cavity, before being ejected further into the cavity. A similar but opposite behaviour is observed in the vicinity of the cooled wall. Figure 2 shows the non-dimensional time interval needed for the average Nusselt number on the heated wall to stabilise statistically. This time scale depends on the Rayleigh number and in all cases is in the order of $\sim 1000$ dimensionless time units.

Fig. 2 Normalised average Nusselt number on the heated wall as a function of non-dimensional time. The normalisation is done with respect to the average Nusselt number on the heated wall, after the flow became statistically steady.

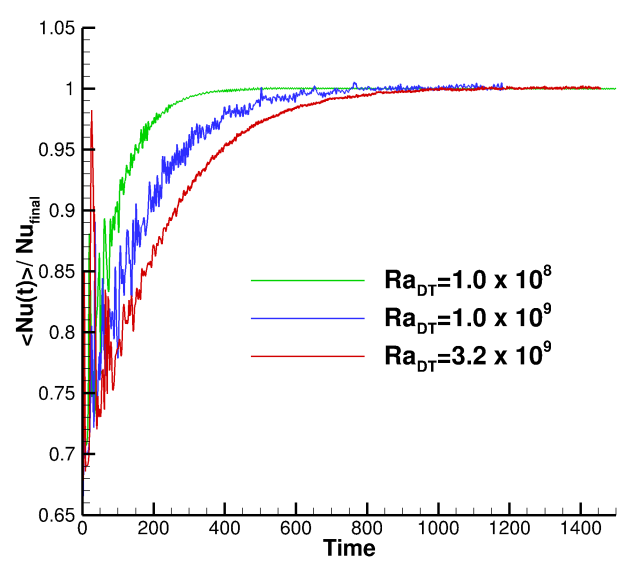

\subsection{Statistics}

After the initial transient stage, the flow becomes statistically steady, with stratified temperature at the center of the cavity and most of the dynamics contained at the top-left and bottom-right corners of the cavity. Figure 3 shows a comparison of the isotherms inside the cavity obtained with the 2D and 3D simulations for the two highest Rayleigh number considered here. It is clear that for a Rayleigh number of $1.0 \times 10^{9}$ the differences are contained only on the top and bottom of the 
cavity, while for $3.2 \times 10^{9}$ also in the bulk of the cavity the isotherms no longer overlap. This illustrates the increase of $3 D$ turbulent structures inside the cavity as the Rayleigh number increases.

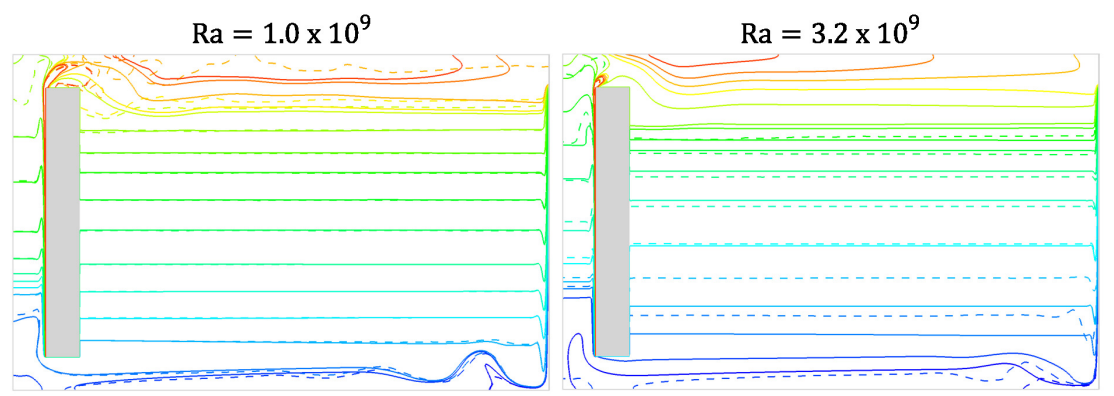

Fig. 3 Comparison of the time-averaged isotherms of $2 D$ (dashed) and $3 D$ (solid) simulations, for $R a=1.0 \times 10^{9}$ (left) and $3.2 \times 10^{9}$ (right).

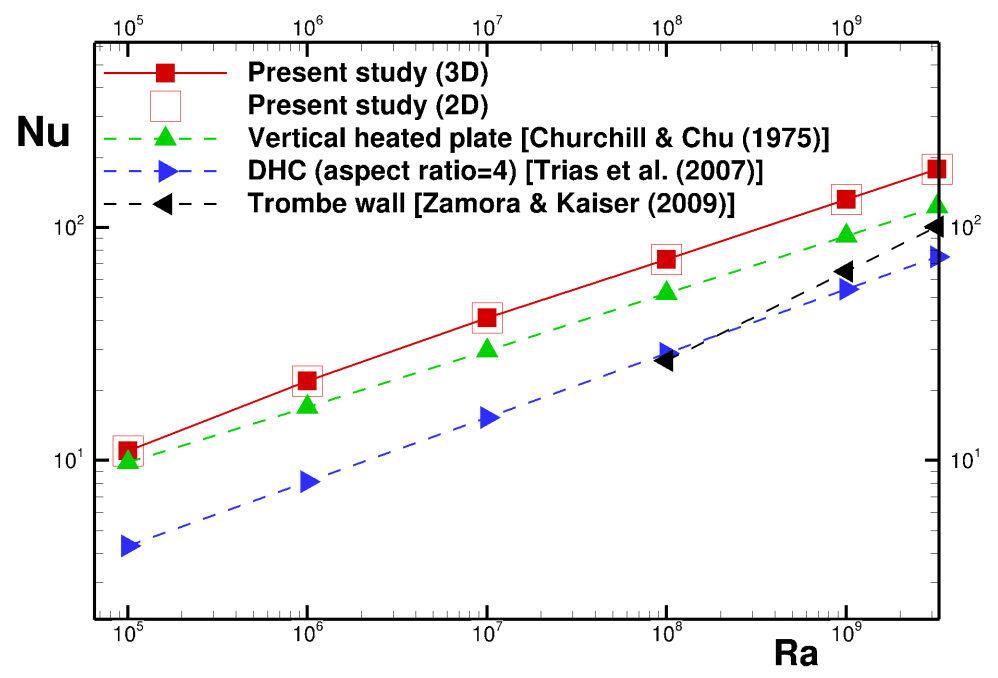

Fig. 4 Predicted Nusselt numbers of the heated wall as a function of Rayleigh numbers compared to similar configurations. For high $R a$ the results can be well approximated with a scaling law $N u=0.518 \times R a^{0.268}$.

Moreover, as shown in figure 4 , the $2 D$ and $3 D$ predictions of the average Nusselt number on the heated wall are very similar in the entire $R a$ range studied. This is explained by the fact that the highest Nusselt numbers are observed on the bottom of the heated wall, where the flow is laminar, while the contribution of the turbulent flow on the top of the heated wall is much less. A strong evidence for scaling of the 
Nusselt number with respect to $R a$ numbers is observed. The calculated correlation $N u=0.518 \times R a^{0.268}$ is also an indication of the similarity between the $2 D$ and $3 D$ Nusselt predictions, since the exponent 0.268 is much closer to the $1 / 4$ laminar scaling than the $1 / 3$ turbulent scaling [7]. Additionally, as shown in figure 4 , the heat transfer rate of the heated wall is consistently higher than that reported for similar configurations such as differentially heated cavities, vertical heated plates and Trombe walls, illustrating its potential for intensified heat transfer.

\section{Conclusions}

The study of the flow inside the asymmetrically heated cavity, revealed that $\sim 1000$ non-dimensional time units are needed for the flow to become statistically steady. The differences between $2 D$ and $3 D$ results become more intense with increasing Rayleigh numbers, but Nusselt numbers as predicted from the 2D and 3D simulations are similar for all Rayleigh numbers studied. Finally the flow exhibits higher Nusselt numbers than other relevant configurations, with a scaling close to the laminar one.

\section{References}

1. Puangsombuta, W., Hirunlabha, J., Khedarib, J., Zeghmatic, B. and Win, M.M. : Enhancement of natural ventilation rate and attic heat gain reduction of roof solar collector using radiant barrier, J. Fluid Mech., 42(6), 2218-2226 (2007).

2. Zamora, B. and Kaiser, A. S. : Thermal and dynamic optimization of the convective flow in Trombe Wall shaped channels by numerical investigation, Heat and Mass Transfer, 45(11), 1393-1407 (2009).

3. Kaloudis, E., Grigoriadis, D.G.E., Papanicolaou, E. and Panidis, T. : Large eddy simulations of turbulent mixed convection in the charging of a rectangular thermal storage tank, Int. J. of Heat and Fluid Flow, 44, 776-791 (2013).

4. Lai, M. C., and Peskin, C. S. : An immersed boundary method with formal second-order accuracy and reduced numerical viscosity, Journal of Computational Physics, 160(2), 705$719,(2000)$.

5. Maystrenko, A., Christian Resagk, and Thess, A. : Structure of the thermal boundary layer for turbulent Rayleigh-Bnard convection of air in a long rectangular enclosure, Physical Review E - Statistical, Nonlinear and Soft Matter Physics, 75(6), 066303, (2007).

6. Shishkina, O., Stevens, R.J.A.M., Grossmann, S. and Lohse, D. : Boundary layer structure in turbulent thermal convection and its consequences for the required numerical resolution, New Journal of Physics, 12(7), 075022, (2010).

7. Churchill, S.W., and Chu, H.H. : Correlating equations for laminar and turbulent free convection from a vertical plate, Int. J. of Heat and Mass Transfer, 18(11), 1323-1329 (1975).

8. Trias, F. X., Soria M., Oliva A., and Prez-Segarra C. D.: Direct numerical simulations of twoand three-dimensional turbulent natural convection flows in a differentially heated cavity of aspect ratio 4,Journal of Fluid Mechanics, 586, 259-293, (2007) . 